Bull. Mater. Sci., Vol. 16, No. 2, April 1993, pp. 109-116. (C) Printed in India.

\title{
Effect of bismuth replacement at barium site in $\mathrm{Y}-\mathrm{Ba}-\mathrm{Cu}-\mathrm{O}$ system
}

\author{
RAM BILAS \\ Department of Physics, Indian Institute of Technology, Kanpur 208016, India \\ MS received 2 June 1992; revised 31 December 1992
}

\begin{abstract}
X-ray diffraction, electron paramagnetic resonance, microwave absorption and resistivity measurements were carried out on $\mathrm{YBa}_{2-x} \mathrm{Bi}_{x} \mathrm{Cu}_{3} \mathrm{O}_{7}(0 \leqslant x \leqslant 0.5)$ superconductors in order to study the effect of bismuth on the structural and superconducting properties. $\mathrm{A}$ decrease in sintering temperature increased the amount of impurity phase. Transition from an orthorhombic (superconducting) phase to tetragonal structure produced no significant change in EPR zero-field signal at liquid nitrogen temperature (LNT). The non-resonance signal height decreased on higher concentration of bismuth. Sintered $\mathrm{YBa}_{2-x} \mathrm{Bi}_{x} \mathrm{Cu}_{3} \mathrm{O}_{7}$ superconductor had a strong EPR zero-field signal at LNT. We have evaluated particle size from XRD and EPR studies. The average particle size was about $0.4 \mu \mathrm{m}$.
\end{abstract}

Keywords. Oxide superconductor; bismuth impurity; microwave absorption.

\section{Introduction}

The discovery of $\mathrm{La}-\mathrm{Sr}-\mathrm{Cu}-\mathrm{O}$ and $\mathrm{Y}-\mathrm{Ba}-\mathrm{Cu}-\mathrm{O}$ oxide superconductor with $T_{c}$ up to $90 \mathrm{~K}$ (Bednorz and Muller 1986; Kiloyne and Cywinski 1987; Muromachi et al 1987; Wu et al 1987; Iqbal et al 1988) has aroused a lot of interest in $\mathrm{Y}-\mathrm{Ba}-\mathrm{Cu}-\mathrm{O}$ superconductor. Bismuth introduced into the $\mathrm{Y}-\mathrm{Ba}-\mathrm{Cu}-\mathrm{O}(1-2-3)$ system to partially replace yttrium (Yang 1990) was reported to have improved grain contact by forming flux during sintering. It was used as an oxide additive in the $\mathrm{Y}-\mathrm{Ba}-\mathrm{Cu}-\mathrm{O}$ system (Jung 1988; Takeyuki et al 1988) which possibly resulted in liquid phase sintering. It was also reported that the partial $\mathrm{Bi}$ substitution had an advantage, that $\mathrm{Bi}_{2} \mathrm{O}_{3}$ seemed to perform the function as an internal catalyst which favoured the growth of superconducting phase. Moreover, it is well known that bismuth oxide was used earlier as a nucleating agent in ceramic materials. The replacement of copper site by $\mathrm{Bi}$ in $\mathrm{Y}-\mathrm{Ba}-\mathrm{Cu}-\mathrm{O}$ system was also investigated earlier (Yang et al 1990). The present investigation is concerned with partial replacement of barium site by bismuth to study the effect of $\mathrm{Bi}$ on structural and superconducting properties of $\mathrm{YBa}_{2-x} \mathrm{Bi}_{x} \mathrm{Cu}_{3} \mathrm{O}_{7-s}$ $(0 \leqslant x \leqslant 0.5)$ high $T_{c}$ superconductors.

\section{Experimental}

The samples were synthesized by the standard solid-state reaction technique. The constituents were weighed (wt.\%) from powders of yttrium oxide $\left(\mathrm{Y}_{2} \mathrm{O}_{3}\right)$, barium carbonate $\left(\mathrm{BaCO}_{3}\right)$, bismuth oxide $\left(\mathrm{Bi}_{2} \mathrm{O}_{3}\right)$ and copper oxide $(\mathrm{CuO})$ each of purity $99.9 \%$. They were mixed and ground thoroughly in an agate pestle mortar. The grinding was repeated twice to achieve homogeneity.

After decomposing at $800^{\circ} \mathrm{C} / 12 \mathrm{~h}$ the powder was pressed into pellet-form by applying a pressure of 8 tonnes $/ \mathrm{cm}^{2}$. The pellets $(\simeq 1.1 \mathrm{~cm}$ in dia. and $0.2 \mathrm{~cm}$ thick) were sintered in two different conditions. Under the first, some pellet samples were heated 
at $950^{\circ} \mathrm{C} / 12 \mathrm{~h}$ and furnace-cooled to room temperature in the presence of oxygen atmosphere and under the second, some other pellet samples were sintered at $950^{\circ} \mathrm{C}$ for $2 \mathrm{~h}$ and slowly cooled to $600^{\circ} \mathrm{C}$. The samples were then kept at this temperature for $5 \mathrm{~h}$.

XRD patterns of the samples were recorded using Rich-Scifert isodebyflex (model 2002) diffractometer with chromium $\left(\mathrm{CrK}_{\alpha}\right)$ target. EPR spectra were recorded using Varian E-109 EPR spectrometer. The sample $\left(2 \times 2 \times 1 \mathrm{~mm}^{3}\right)$ was kept in a sealed quartz tube and a thermocouple sensor placed in contact with the sample. The temperature was controlled with a variable temperature controller with accuracy of $\pm 0.5 \mathrm{~K}$. The frequency of the resonance cavity was measured using a standard $g$-marker, (DPPH) $g=2 \cdot 0036 \pm 0.0002$. The resistivity was measured using a standard four-probe technique. Knick precision constant current source (model 181) and Keithley nanovoltmeter were used for resistivity measurements.

\section{Results and discussion}

Figure 1 shows X-ray patterns for samples with $x=0.0,0.1,0.2,0.3$ and 0.5 . The system showed orthorhombic nature up to $x=0.2$ doping of $\mathrm{Bi}$ and after that the samples became multiphase for $x=0.3$ and 0.5 . As $x$ increased another group of peaks emerged with increased intensity which was identified as $\mathrm{YBa}_{2} \mathrm{BiO}_{6}$ and $\mathrm{CuO}$ (Yang et al 1990). No impurity peaks other than these three could be found in the patterns. The $\mathrm{YBa}_{2} \mathrm{BiO}_{6}$ phase co-existed with 1-2-3 and varied only in the composition range. When $x=0.5$, the XRD pattern mainly consists of $\mathrm{YBa}_{2} \mathrm{BiO}_{6}$ and $\mathrm{CuO}$ with the 1-2-3 peaks hardly discernible. When bismuth replaces part of $\mathrm{Ba}$ in $1-2-3$ composition a proportional phase of $\mathrm{YBa}_{2} \mathrm{BiO}_{6}$ is formed (Blower and Greeves 1988).

The lattice parameters $\mathrm{a}, \mathrm{b}, \mathrm{c}(950 / 12 \mathrm{~h})$ for this series have been calculated (table 1$)$. With different concentrations of Bi from X-ray diffraction patterns, it appeared that $d$-values increased with substitution of $\mathrm{Bi}$ in the $\mathrm{Ba}$ plane. The orthorhombic phase decreased and tetragonal phase increased at higher $\mathrm{Bi}$ concentrations. The system formed tetragonal structures lowering the superconducting transition $\left(T_{c}\right)$.

The broadening of some of the particular peaks i.e. 103, 005, 123 has been considered. The average grain-size estimated from XRD patterns is given in table 2 . Half-line width depends upon two factors, the particle (grain-size) and the lattice strain (lattice distortion). Normally the grain size of the material is $1 \mu \mathrm{m}$. Thus, line broadening seems to be due to lattice strain as is evident from the lattice parameter as shown in figure 2. The line broadening passes through a maxima around $x=0 \cdot 1$ of $\mathrm{Bi}$ substitution. Thus it seems that substitution of $\mathrm{Bi}$ in $\mathrm{YBa}_{2-x} \mathrm{Bi}_{x} \mathrm{Cu}_{3} \mathrm{O}_{7-s}$ lattice is maxima and corresponds to the maximum incorporation of $\mathrm{Bi}$.

The EPR spectra are shown in figure 3. A small zero-field signal appears at $x=0.2$, after which the compound became multiphase and its signal amplitude zero field decreased. The spectra were recorded in a small piece $(2 \times 2 \times 1 \mathrm{~mm})$ from the compound. The room temperature EPR signal consisted of a peak typical of $s= \pm 1 / 2$ species, showing the $g$-value anisotropy. Since the expected paramagnetic species should arrive from $\mathrm{Cu}^{2+} / \mathrm{Cu}^{3+}$, it is clear from the EPR spectra that dominant valence is $\mathrm{Cu}^{2+}$ from the orthorhombic symmetry $\mathrm{Cu}^{2+}$ which will show the $g$-value anisotropy. The presence of only two resolved components with $g_{\|}=2.0 \pm 0.02$ and 


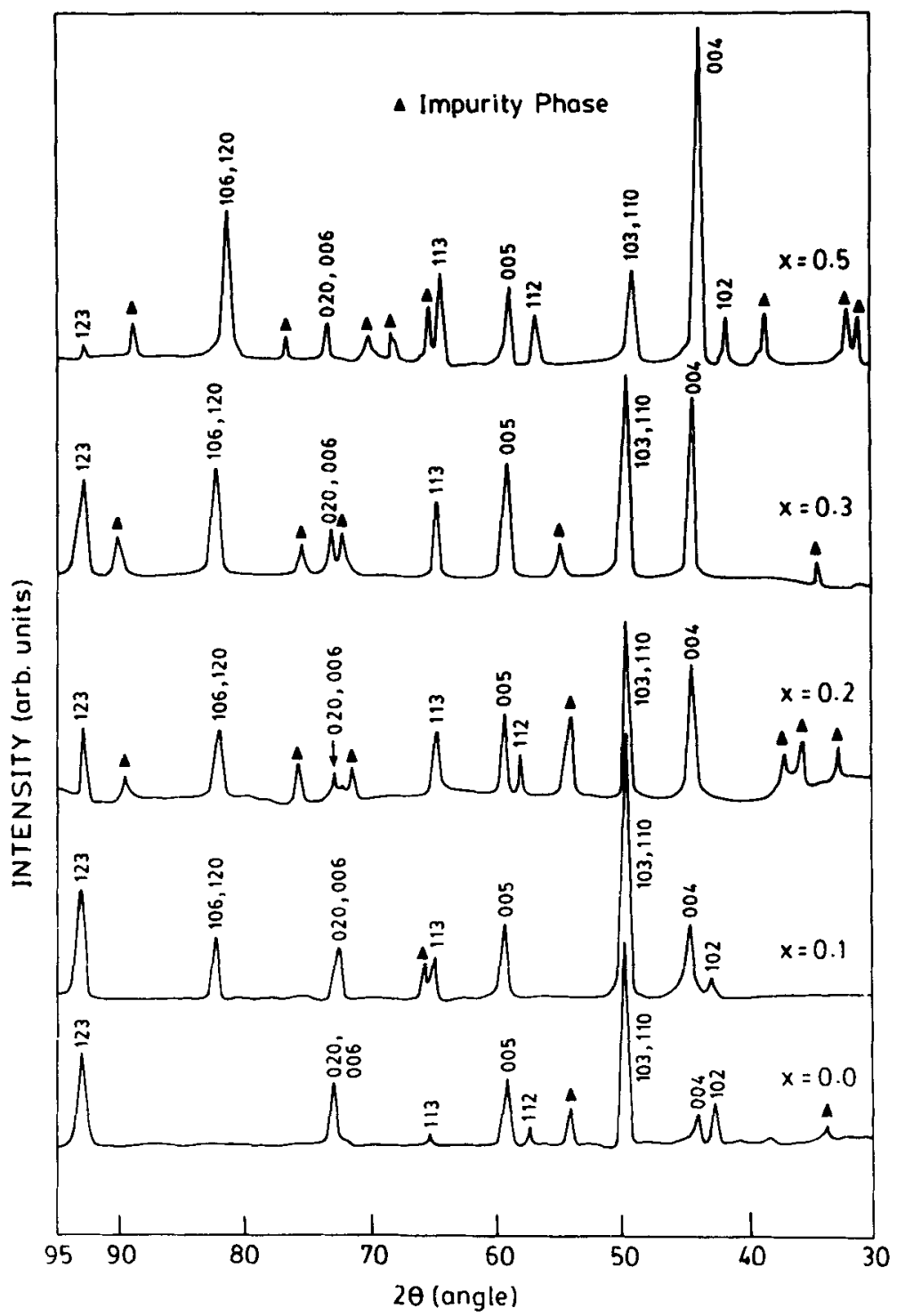

Figure 1. X-ray diffraction patterns of $\mathrm{YBa}_{2-x} \mathrm{Bi}_{x} \mathrm{Cu}_{3} \mathrm{O}_{7-8}(0 \leqslant x \leqslant 0.5)$ samples.

Table 1. Variation of lattice parameters for $\mathrm{YBa}_{2-x} \mathrm{Bi}_{x} \mathrm{Cu}_{3} \mathrm{O}_{7-\delta}(0 \leqslant x \leqslant 0 \cdot 5)$ heat-treated at $950^{\circ} \mathrm{C} / 12 \mathrm{~h}$

\begin{tabular}{ccccc}
\hline$X$ & $a$ & $b$ & $c$ & $v$ \\
\hline 0.0 & 3.80 & 3.87 & 11.67 & 171.70 \\
0.1 & 3.84 & 3.91 & 11.71 & 175.91 \\
0.2 & 3.81 & 3.88 & 11.68 & 172.27 \\
0.3 & 3.85 & 3.92 & 11.72 & 176.96 \\
0.5 & 3.88 & 3.95 & 11.75 & 180.17 \\
\hline
\end{tabular}


Table 2. Average particle size for $\mathrm{YBa}_{2-x} \mathrm{Bi}_{x} \mathrm{Cu}_{3} \mathrm{O}_{7-\delta}$ $(0 \leqslant x \leqslant 0 \cdot 5)$ heat-treated in different conditions.

\begin{tabular}{lccccc}
\hline & \multicolumn{2}{c}{$\begin{array}{c}\text { Particle size }(\mu \mathrm{m}) \\
\left(950^{\circ} \mathrm{C} / 12 \mathrm{~h}\right)\end{array}$} & & \multicolumn{2}{c}{$\begin{array}{c}\text { Particle size }(\mu \mathrm{m}) \\
\left(950^{\circ} \mathrm{C} / 2 \mathrm{~h}+600^{\circ} \mathrm{C} / 5 \mathrm{~h}\right)\end{array}$} \\
\cline { 2 - 3 } \cline { 5 - 6 } & XRD & EPR & & XRD & EPR \\
\hline 0.0 & 0.40 & 0.41 & & 0.31 & 0.41 \\
0.1 & 0.34 & 0.41 & 0.25 & 0.41 \\
0.2 & 0.43 & 0.41 & 0.34 & 0.41 \\
0.3 & 0.33 & - & 0.34 & - \\
0.5 & - & - & 0.38 & - \\
\hline
\end{tabular}

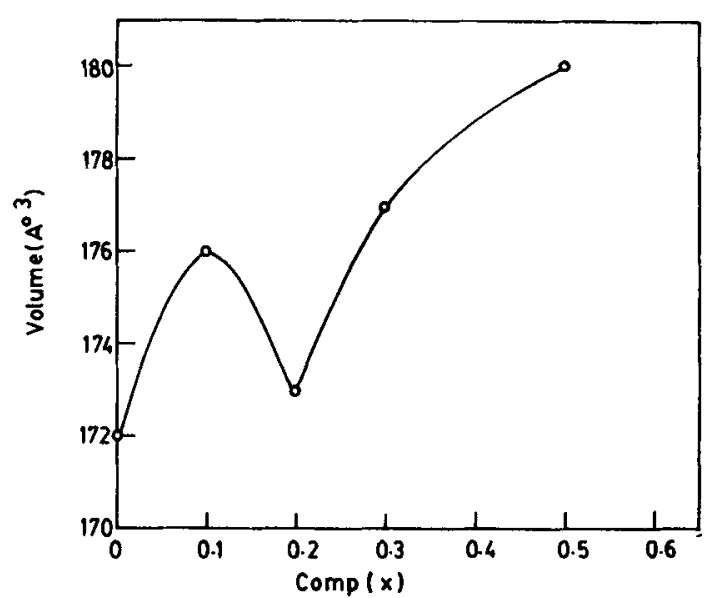

Figure 2. Lattice volume change with increase in $x$-value of the system $\mathrm{YBa}_{2-x} \mathrm{Bi}_{x} \mathrm{Cu}_{3} \mathrm{O}_{7-\delta}$ $(0 \leqslant x \leqslant 0 \cdot 5)$.

$g_{\perp}=2 \cdot 13 \pm 0.02$ further indicates the axial symmetry of $\mathrm{Cu}^{2+}$ environments. The fact that $g_{\|} \leqslant g_{\perp} \leqslant 2$ indicates that ground state orbital of $\mathrm{Cu}^{2+}$ is $\mathrm{d} x^{2}-\mathrm{d} y^{2}$ (Bhat et al 1987; Blazey et al 1987).

Transition from normal to superconducting state takes place between $72 \mathrm{~K}$ and $92 \mathrm{~K}$ and this has also been recorded at LNT temperature (figures 3-6). There is a very strong signal in the superconducting phase at very low field $(0-50 \mathrm{G})$. The estimated EPR spectral parameters are given in table 3 . The samples $x=0.0,0 \cdot 1,0 \cdot 2$ are shown near zero-field microwave absorption signal (figures 4,6 ) but no signal $(x>0 \cdot 3)$ of the system has been observed due to its glassy nature. The average grainsize is estimated from EPR to be $=0.4 \mu \mathrm{m}$. We have compared the particle size determined from XRD and EPR and they are given according to the following formula (Blazey et al 1987) $S=\phi / 2 H_{\mathrm{c} 1}$ where, $\phi$ is $2.0678 \times 10^{-7} \mathrm{G} / \mathrm{cm}^{2}$ and $S$, the loop area.

The average particle size is nearly the same from EPR and XRD (table 2). From resistivity data superconducting transition $\left(T_{c}\right)$ was observed for system $\mathrm{YBa}_{2-x} \mathrm{Bi}_{x} \mathrm{Cu}_{3} \mathrm{O}_{7-s}$ by temperature dependence of resistivity. In spite of irregularities of porosity of the samples, the contact was important for measurement. It appears from the graph that the normal state resistance behaviour is metallic. The deviation 


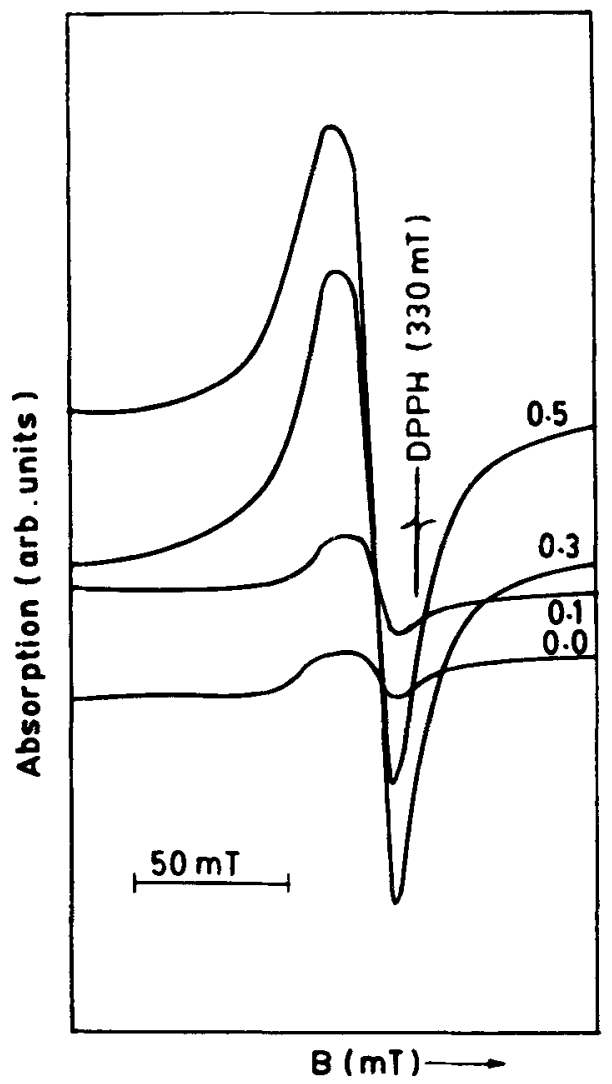

Figure 3. EPR spectra of $\mathrm{YBa}_{2} \mathrm{Cu}_{3} \mathrm{O}_{7}$ : Bismuth doped system at room temperature. (The samples were prepared at $950^{\circ} \mathrm{C} / 12 \mathrm{~h}$ in presence of oxygen).

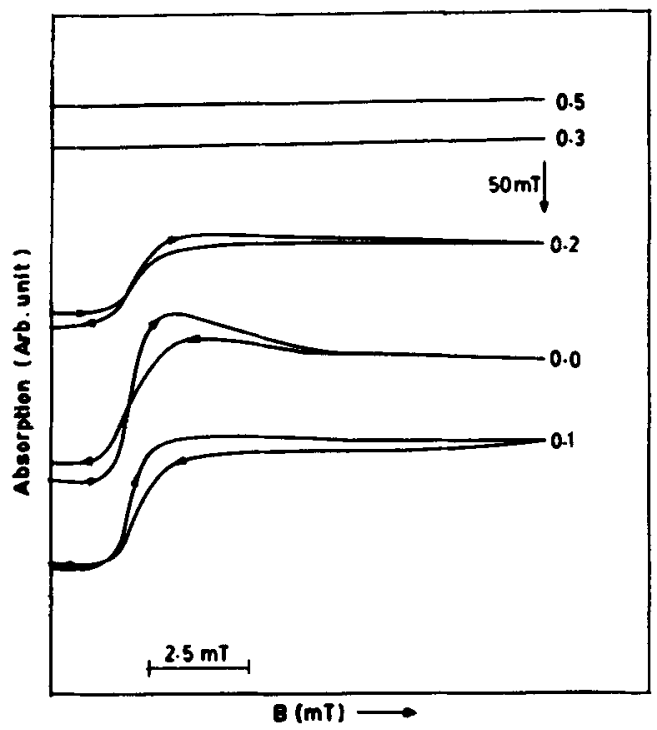

Figure 4. Low field absorption spectra of $\mathrm{YBa}_{2-x} \mathrm{Bi}_{x} \mathrm{Cu}_{3} \mathrm{O}_{7-\delta}(0 \leqslant x \leqslant 0 \cdot 5)$ at liquid nitrogen temperature for forward and reverse sweeps. (The $\mathrm{Bi} w \mathrm{t} \%$ are shown against the spectra $\left.\left(950^{\circ} \mathrm{C} / 12 \mathrm{~h}\right)\right)$. 


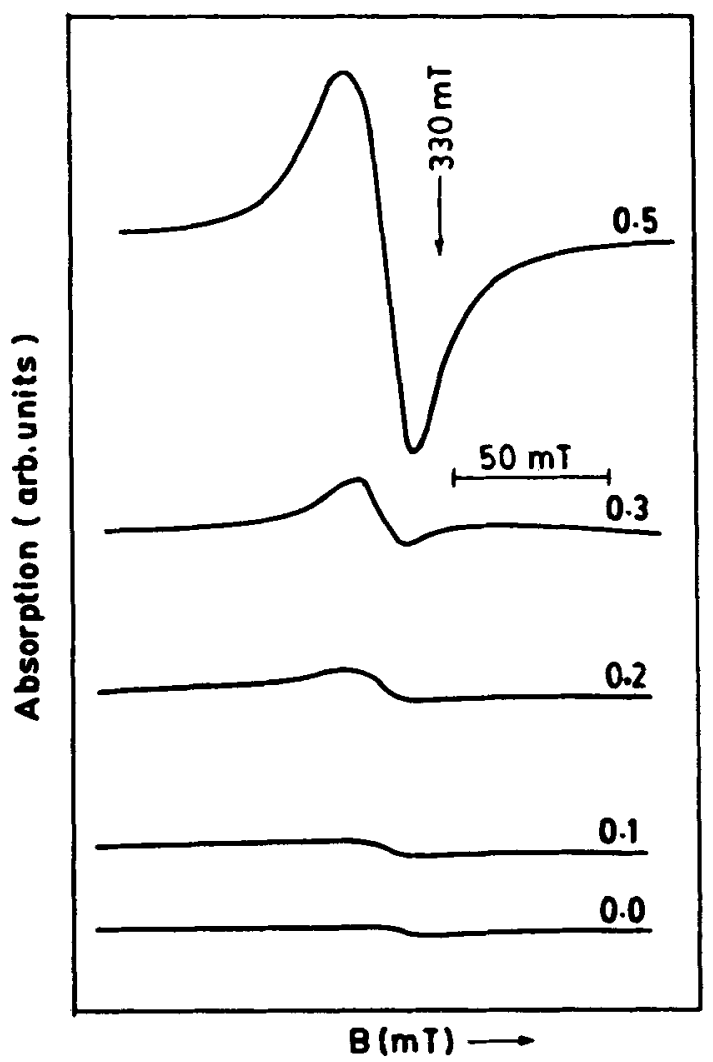

Figure 5. EPR spectra of $\mathrm{YBa}_{2} \mathrm{Cu}_{3} \mathrm{O}_{7}$ : $\mathrm{Bi}$ doped system at room temperature. The samples were prepared at $950^{\circ} \mathrm{C} / 2 \mathrm{~h}+600^{\circ} \mathrm{C} / 5 \mathrm{~h}$ in presence of oxygen.

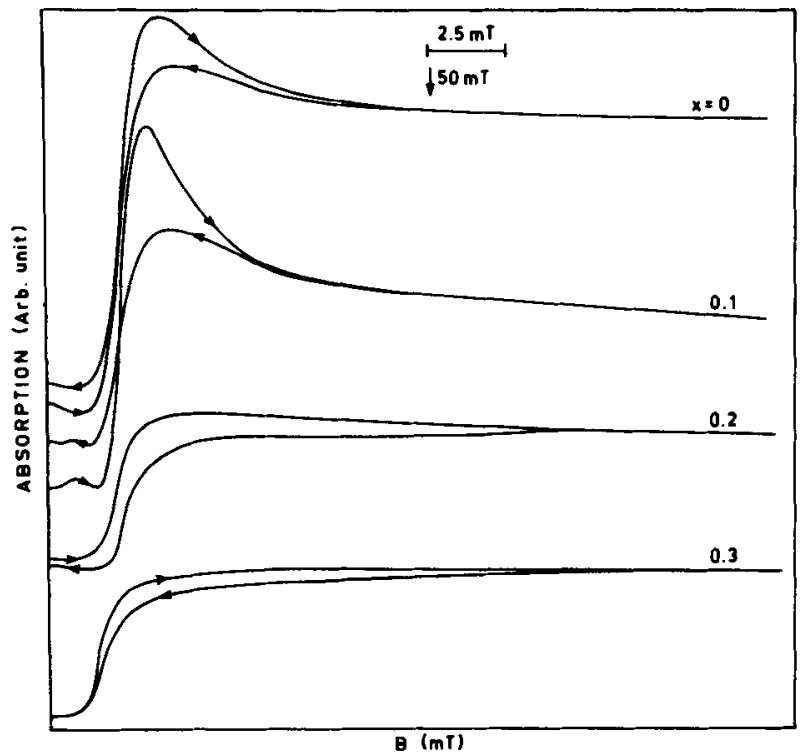

Figure 6. Low field absorption spectra of $\mathrm{YBa}_{2} \mathrm{Cu}_{3} \mathrm{O}_{7}$ Bi doped samples at liquid nitrogen temperature for forward and reverse sweeps. (The $\mathrm{Bi}$ wt \% are shown against the spectra (samples were prepared by $950^{\circ} \mathrm{C} / 2 \mathrm{~h}+600^{\circ} \mathrm{C} / 5 \mathrm{~h}$ in presence of oxygen)). 


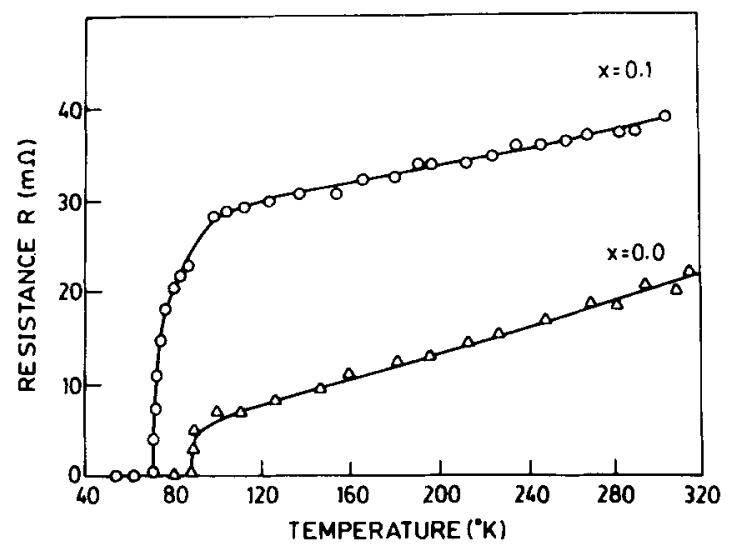

Figure 7. The typical plots of temperature dependence of resistance of pure and $0.1 \mathrm{wt} \%$ Bi doped of $\mathrm{YBa}_{2} \mathrm{Cu}_{3} \mathrm{O}_{7}$

Table 3. EPR and non-resonant microwave absorption data of $\mathrm{YBa}_{2-x} \mathrm{Bi}_{x} \mathrm{Cu}_{3} \mathrm{O},-\delta(0 \leqslant x \leqslant 0 \cdot 5)$.

\begin{tabular}{lccc}
\hline$X$ & $\begin{array}{c}\mathrm{H}_{\text {o peak }} \\
(\mathrm{G})\end{array}$ & $\begin{array}{c}\text { Resonance absorption } \\
\text { signal }(\mathrm{cm})\end{array}$ & $\begin{array}{c}\text { Non-resonance } \\
\text { absorption signal }(\mathrm{cm})\end{array}$ \\
\hline $0 \cdot 0$ & 25 & 0.00 & 6.4 \\
$0 \cdot 1$ & 25 & 0.22 & 5.0 \\
0.2 & 25 & 0.31 & 3.6 \\
0.3 & - & 0.40 & - \\
0.5 & - & 2.14 & - \\
\hline
\end{tabular}

towards zero from the linear dependence in resistivity was observed near $T_{c}$. It was also observed that the superconducting transition temperature decreased with $\mathrm{Bi}$ concentration.

\section{Conclusion}

New composition of high $T_{c}$ superconductor $\mathrm{YBa}_{2-x} \mathrm{Bi}_{x} \mathrm{Cu}_{3} \mathrm{O}_{7-\delta}$ with $x \leqslant 0.5$ have been prepared by the usual ceramic method. The superconducting phase and crystallites exhibited very small average particle size i.e. $0.4 \mu \mathrm{m}$, from EPR as well as X-ray diffraction studies.

\section{Acknowledgements}

The author is grateful to Dr Prem Chand and Mr S V Sharma for valuable suggestions.

\section{References}


Blazey K W, Muller K A and Bednorz J G 1987 Phys. Rev. B36 7241

Iqbal Z, Eckhard H, Reidinger F, Bose A, Barry J C and Ramakrishna B L 1988 Phys. Rev. B38 859

Jung J, Frank J P, Miner Wa A and Mohamed M A K 1988 Phys. Rev. B37 7510

Kiloyne S H and Cywinski R 1987 J. Phys. D. (Appl. Phys.) 201327

Muromachi E T, Uchida Y, Matsui Y and Kato K 1987 Jpn. J. Appl. Phys. 26 L476; 26619

Takeyuki S, Tsutomu Y, Akinori K, Sashi M and Hisashi S 1988 J. Mater. Sci. Lett. 7926

Wu M K, Ashburn J R, Torng C J, Hor P H, Mang R L, Gao L, Huang Z J, Wamg Y Q and Chu C W 1987 Phys. Rev. Lett. $\mathbf{5 8} 908$

Yang N, Liu R S, Wang W N and Wu P P 1990 J. Mater. Sci. 254758 